\title{
Enhancement of two-photon emission in photonic crystals
}

\author{
Przemyslaw Markowicz, Christopher Friend, Yuzhen Shen, Jacek Swiatkiewicz, and Paras N. Prasad \\ The Institute for Lasers, Photonics and Biophotonics, Departments of Chemistry and Physics, \\ State University of New York at Buffalo, Buffalo, New York 14260
}

Ovidiu Toader and Sajeev John

Department of Physics, University of Toronto, 60 St. George Street, Toronto, Ontario M5S 1A7, Canada

Robert W. Boyd

The Institute of Optics, University of Rochester, Rochester, New York 14627

Received July 31, 2001

\begin{abstract}
We report the influence of photonic stopgaps on two-photon excited emission from highly efficient nonlinear chromophores infiltrated into high-quality photonic crystals. We have observed a sharp decrease (filter effect) in emission within the frequency range and direction of the stopgap as well as sharp enhancement of the two-photon excited emission associated with the stopgap's edge. This effect may be important for the development of low-threshold upconversion lasers. (C) 2002 Optical Society of America

OCIS codes: $190.7220,190.4400$.
\end{abstract}

Three-dimensional periodic dielectric structures in which the refractive index varies on length scales of the order of optical wavelengths have recently attracted much theoretical and experimental attention. ${ }^{1-3}$ An especially interesting class of such structures are photonic bandgap crystals. For the range of frequencies defined by the photonic bandgap, no light can propagate through the crystal in any direction. Instead, light in this frequency range is localized. ${ }^{4-6}$ This localization has a significant effect on the spontaneous emission of excited molecules placed inside the photonic crystal. ${ }^{7,8}$ If the transition frequency of excited molecules is adjusted to the frequency range of the gap, spontaneous emission of light into unwanted modes can be completely inhibited. This effect may allow for the development of thresholdless lasers. ${ }^{8-10}$

A number of experiments with one-photon excitation of dyes incorporated into ordered ${ }^{11-13}$ and disordered ${ }^{14}$ photonic structures, which act as lasing materials, have been undertaken. In the case of ordered structures, the range of order associated with the crystal building blocks and the refractive index determine the optical properties of these structures. Currently, polystyrene particles are the building blocks with the highest refractive index, $\sim 1.6$, permitting the production of photonic crystals with long-range particle order. Although the scattering properties of the crystals are still weak, they are sufficiently strong to allow us to observe the influence of the crystals on two-photon emission.

In this Letter we report experimental results of the modification of two-photon excited, upconverted emission spectra from a highly efficient dye incorporated into photonic crystals that exhibit stopgaps ranging in size from $1 \%$ to $3 \%$ of the gap center frequency. The photonic crystals were fabricated from polystyrene spheres with a diameter of $200 \mathrm{~nm}$ and then infiltrated with dye. Coumarin 503 dye was chosen for the infiltration because the emission spectrum of this dye fits into the stopgap of the transmission spectrum of the polystyrene photonic crystal and because its highfluorescence quantum efficiency [0.84 (Ref. 15)] leads to mostly radiative decay.

The method introduced by Park and $\mathrm{Xia}^{16}$ was applied for the crystallization of polystyrene nanoparticles. The transmission spectra of photonic structures were measured with the aid of a Biorad MRC-1024 confocal scanning microscope mounted upon a Nikon Eclipse-800 upright microscope. The side output port of the microscope was connected to a spectrometer (HoloSpec, Kaisar Optical Systems, Inc.) with a multimode fiber. The white light was focused upon the surface of the sample, and the transmitted light was collected by a Nikon Plan $10 \times$ objective with a N.A. of 0.25 . We observed the central wavelengths of the stopgaps at $\sim 500$ and $\sim 525 \mathrm{~nm}$ for polystyrenemethanol and polystyrene-dimethyl sulfoxide (DMSO) crystals, respectively (Fig. 1). Sharp attenuation with transmission larger than $80 \%$ outside the gap suggests long-range particle order in the crystals. The

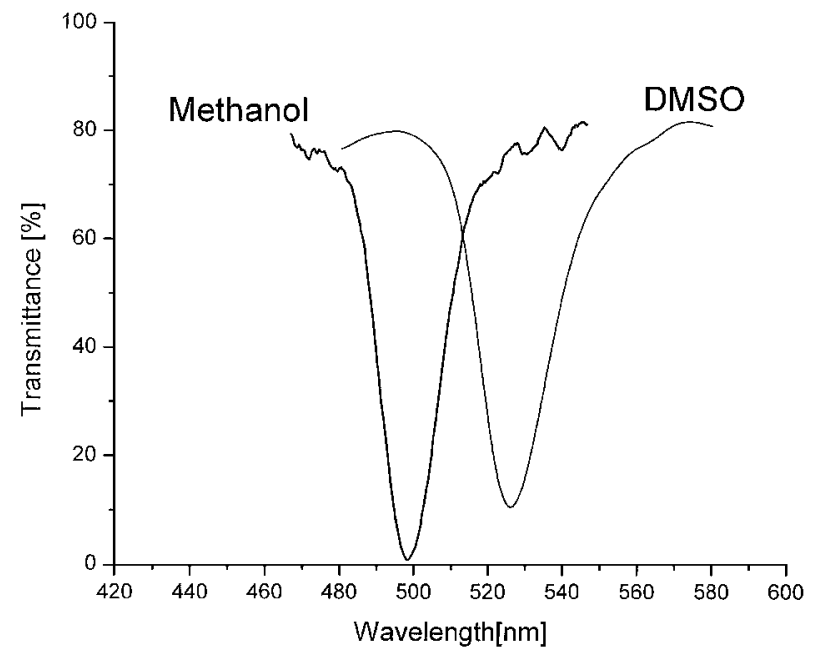

Fig. 1. Transmission spectra of polystyrene-methanol and polystyrene-DMSO assemblies (without dye). 
photonic band structure of the polystyrene-methanol crystal is shown in Fig. 2.

Scanning-electron microscope images of a dried sample (not shown) confirmed the long-range particle order in the crystals and the fact that polystyrene particles crystallize into an $f c c$ structure with the (111) face parallel to the surface. We illuminated the surface with light at normal incidence. Because the refractive-index contrast is small, the stopgap position is accurately approximated by the Bragg equation ${ }^{16}$

$$
\lambda_{111}=2 n_{\mathrm{eff}} d_{111},
$$

where $d_{111}$ is the spacing between (111) planes in the $f c c$ crystal and $n_{\text {eff }}=\lim _{k \rightarrow 0}(c k / \omega)$ is the effective refractive index obtained by considering the long-wavelength limit of photon dispersion relation $\omega(k)$ and where $c$ is the speed of light. The refractive indices of polystyrene, methanol, and DMSO are $n_{\mathrm{pol}}=1.6$, $n_{\text {meth }}=1.33$, and $n_{\text {DMSO }}=1.48$, respectively.

There was good agreement between the theoretical calculations of the position of the stopgap $(501.8 \mathrm{~nm})$ and experimental results $(498 \mathrm{~nm})$ for the polystyrene-methanol photonic crystal. Similar calculations performed for the polystyrene-DMSO structure showed a difference of $10 \mathrm{~nm}$ between the theoretical and the experimental results, which were 515 and $525 \mathrm{~nm}$, respectively. The reason why the gap was shifted does not seem to have had any influence on our two-photon experiments because the particles were still well ordered.

Photonic crystals with the range of order similar to those whose transmission spectra are presented in Fig. 1 were used in our two-photon experiments. After the polystyrene particles had been assembled during fabrication, the sample was infiltrated with Coumarin 503 dissolved in methanol or in DMSO. The concentrations of Coumarin 503 in methanol and in DMSO were $2.9 \times 10^{-2}$ and $11.77 \times$ $10^{-2} \mathrm{M}$, respectively. Then the sample was illuminated with laser light (described below) and the two-photon excited upconverted emission spectrum was measured.

We obtained two-photon excited fluorescence spectra of the dye in a photonic crystal by exciting the dye with a mode-locked Ti:sapphire laser at a wavelength of $800 \mathrm{~nm}(80 \mathrm{fs})$. The direction of the laser light was perpendicular to the surface of the sample. We collected spectra in the reflection mode (Nikon Plan 10X objective). Laser light with a power of $\sim 20 \mathrm{~mW}$ was focused onto the sample. The diameter of the focal spot size was $\sim 5 \mu \mathrm{m}$. Because the scanning-electron microscope images of the crystal surface indicated that the crystal domains extended over an area of at least a few hundred square micrometers, it was possible to confine the focused spot to a single crystal domain.

For two-photon excited emission spectra of Coumarin 503 in our structure we noticed that, besides filter effects similar to those reported for transmission of external plane waves, ${ }^{13,17}$ a sharp maximum can also appear (Fig. 3). Although the concentration of solvated dye molecules was relatively high, we did not observe any changes in spectral shape attributable to the dipole-dipole transfer of excitation. The emission spectrum of the dye solution, without a photonic crystal, overlapped well with the rescaled spectrum taken for the solution with a 100-times lower dye concentration. In both spectra the sharp maximum did not appear. The maximum seems to be a result of amplification of light inside the photonic crystal. The position of the sharp maximum depends on the position of the stopgap. Outside the stopgap the shapes of the emission spectra from Coumarin 503 were similar for the polystyrene-methanol and polystyrene-DMSO structures (Fig. 3). Both spectra also overlapped with the rescaled reference spectra outside the stop-gap region. The reference spectrum was measured for Coumarin 503 placed in a cell with the same thickness as the polystyrene structure $(8 \mu \mathrm{m})$.

In our experiment, in contrast to those presented in other reports,${ }^{12,13}$ the attenuation and amplification of light in emission spectra exist simultaneously. To check the position of the maximum with respect to the stopgap more precisely, we performed the following experiment: before the two-photon excited upconverted emission spectrum of Coumarin 503 was measured, the transmission spectrum of exactly the same region of

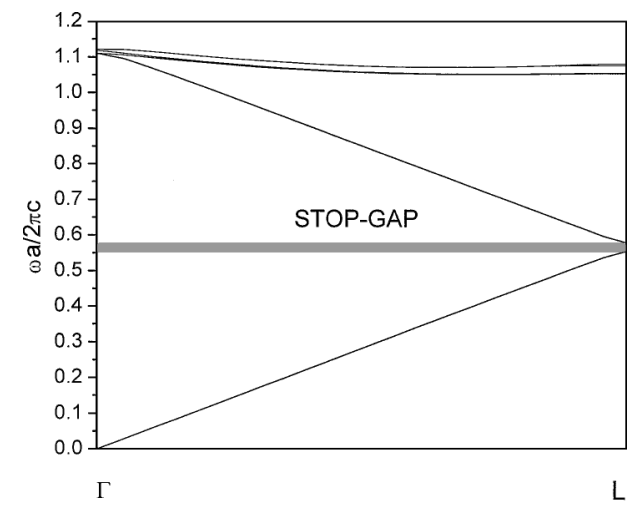

Fig. 2. Band structure of a polystyrene-methanol $\left(n_{\text {eff }}=1.529\right.$; stopgap, $\left.2.96 \%\right)$ photonic crystal. Filling factor, $74 \%$. $a$ is the lattice constant.

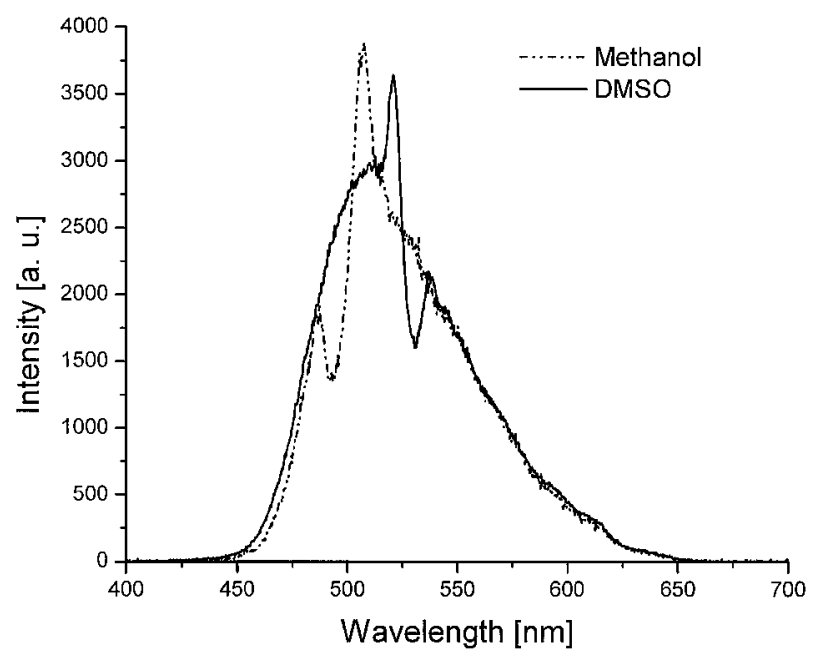

Fig. 3. Two-photon excited upconverted emission spectra of Coumarin 503 in polystyrene-methanol and polystyrene-DMSO photonic structures. 


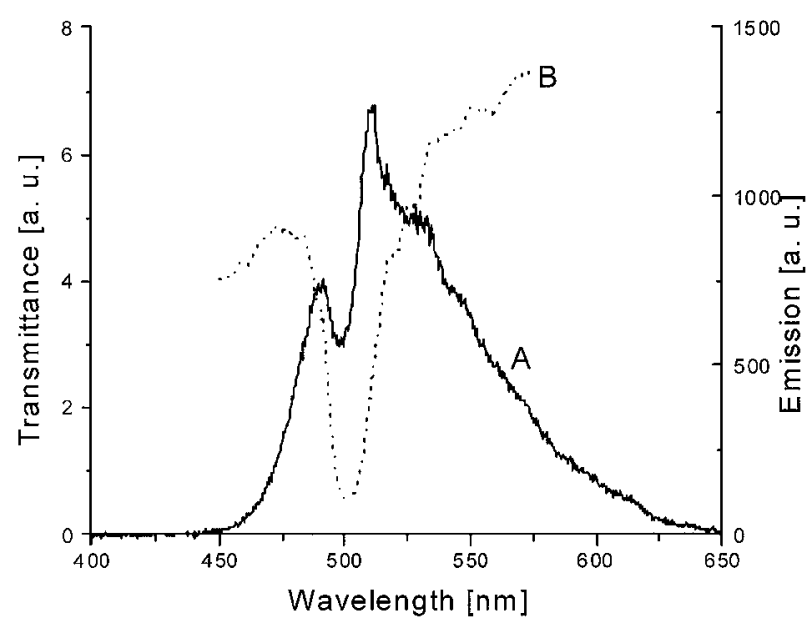

Fig. 4. A, Two-photon excited emission spectra and $\mathrm{B}$, linear transmission spectrum measured at the same spot in polystyrene-Coumarin 503 in a methanol photonic crystal.

the sample was recorded. The results of that experiment are shown in Fig. 4. The amplification in the two-photon emission spectra appeared at the edge of the attenuation in the transmission spectra of external plane waves. This means that the amplification of two-photon excited upconverted emission took place at the edge of the $(1,1,1)$ stopgap.

In our case, the threshold for laser emission was not yet reached, as the magnitude of the maximum was relatively small. However, the influence of the photonic stopgap on the emission spectrum is noticeable because the maximum did not appear in the reference spectrum. It is exciting to see that, even with the current refractive-index contrast, the amplification of two-photon emission was observed. Therefore more dramatic enhancements may be expected in more strongly scattering photonic crystals. ${ }^{18}$

Although the lasing threshold was not reached, it is useful to think of our results in terms of distributed feedback in three-dimensional structures. Conditions for the propagation of electromagnetic waves in a periodic medium with absorption and gain in onedimensional distributed-feedback lasers were reported by Kogelnik and Shank. ${ }^{19}$ The positive feedback in these structures appears to be due to elastic Bragg scattering.

In our structure the feedback was realized as a result of coupling between the incident field and the counterpropagating wave created by Bragg reflection from the (111) crystal planes. However, in our case, in contrast with the Kogelnik-Shank theory ${ }^{19}$ there was only one significant maximum. The presence of only one maximum in an emission spectrum can be due to the gain characteristics of the dye medium. Alternatively, maxima in the emission spectra can appear as a result of the enhanced fluorescence that is predicted to occur near the photonic band edge ${ }^{20}$ or can arise from defects in a photonic crystal.
In conclusion, we have demonstrated the influence of well-ordered photonic crystals on the two-photon excited upconverted emission of highly efficient nonlinear dye. Attenuation and an enhancementlike maximum in the emission spectrum were observed. The attenuation can be understood as a pure filter effect in a way similar to that of the transmission spectrum. The maximum can be interpreted in terms of a distributed-feedback mechanism that appeared as a result of elastic Bragg scattering at the stopgap's edge. Our results suggest that low-threshold upconverted lasing may be attained near the band edges of strongly scattering photonic crystals in which the corresponding feedback effects are much stronger than in other crystals.

This research was supported by U.S. Air Force Office of Scientific Research (USAFOSR) grant F4962000-1-0064, USAFOSR Defense University Research Initiative on Nanotechnology grant F49620-01-1-0358 to the authors in Buffalo, and USAFOSR grant F49620-00-1-0061 to the author in Rochester. P. Markowicz's e-mail address is ppm@acsu.buffalo.edu.

\section{References}

1. A. Blanco, E. Chomski, S. Grabtchak, M. Ibisate, S. John, S. W. Leonard, C. Lopez, F. Meseguer, H. Miguez, J. P. Mondia, G. A. Ozin, O. Toader, and H. M. van Driel, Nature 405, 437 (2000).

2. K. Bush and S. John, Phys. Rev. E 58, 3896 (1998).

3. S. G. Johnson and J. D. Joannopoulos, Appl. Phys. Lett. 77, 3490 (2000).

4. S. John, Phys. Rev. Lett. 53, 2169 (1984).

5. S. John, Phys. Today 44(5), 32 (1991).

6. S. John, Phys. Rev. Lett. 58, 2486 (1987).

7. V. P. Bykov, Sov. J. Quantum Electron. 4, 861 (1975).

8. E. Yablonovitch, Phys. Rev. Lett. 58, 2059 (1987).

9. Y. Yamamoto and R. E. Slusher, Phys. Today 46(6), 66 (1993).

10. S. John, in Photonic Band Gap Materials, C. M. Soukoulis, ed. (Kluwer Academic, Dordrecht, The Netherlands, 1996), p. 563.

11. V. N. Bogomolov, S. V. Gaponenko, I. N. Germanenko, A. M. Kapitonov, E. P. Petrov, N. V. Gaponenko, A. V. Prokofiev, A. N. Ponyavina, N. I. Silvanovich, and S. M. Samoilovich, Phys. Rev. E 55, 7619 (1997).

12. K. Yoshino, S. Tatsuhara, Y. Kawagishi, M. Ozaki, A. A. Zakhidov, and Z. V. Vardeny, Appl. Phys. Lett. 74, 2590 (1999).

13. M. Megens, J. E. G. J. Wijnhoven, A. Lagendijk, and W. L. Vos, J. Opt. Soc. Am. B 16, 1403 (1999).

14. N. Lawandy, R. Balachandran, A. Gomes, and E. Sauvain, Nature 368, 436 (1994).

15. A. N. Fletcher and D. E. Bliss, Appl. Phys. 16, 289 (1978).

16. S. H. Park and Y. Xia, Langmuir 15, 266 (1999).

17. T. Yamasaki and T. Tsutsui, Appl. Phys. Lett. 72, 1957 (1998).

18. N. Vats and S. John, Phys. Rev. A 58, 4168 (1998).

19. H. Kogelnik and C. V. Shank, J. Appl. Phys. 43, 2327 (1972).

20. M. D. Tocci, M. Scalora, M. J. Bloemer, J. P. Dowling, and Ch. M. Bowden, Phys. Rev. A 53, 2799 (1996). 